\title{
Vaginoplasty using Inguinal Flap in a Tertiary Care Center
}

\author{
Zenilda Vieira Bruno ${ }^{1}$, Milena Sayuri Saraiva Ikeda ${ }^{2}$, Marcelo Praxedes Monteiro \\ Filho $^{3}$, Paula Bruno Monteiro ${ }^{4}$, Nívea Adriano de Santana e Santos ${ }^{5}$, Maria Vieira de \\ Lima Saintrain ${ }^{6}$ \\ ${ }^{1}$ Gynecology and obstetrics professor at the Federal University of Ceará (UFC), Fortaleza, Ceara, \\ Brazil \\ ${ }^{2}$ Medical Student of the Federal University of Ceará (UFC), Fortaleza, Ceara, Brazil \\ ${ }^{3}$ Medical Student of the University of Fortaleza (UNIFOR), Fortaleza, Ceara, Brazil \\ ${ }^{4}$ Biomedical. Master student of the Department of Public Health of the Federal University of Ceará \\ (UFC), Scholarship CNPq \\ ${ }^{5}$ Master in Public Health, University of Fortaleza (UNIFOR), Fortaleza, Ceara, Brazil \\ ${ }^{6}$ Master's Professor of Public Health, University of Fortaleza (UNIFOR), Fortaleza, Ceara, Brazil.
}

\begin{abstract}
:
Introduction: The neovagina consists of the construction of a vagina in patients with its complete or partial absence. Mayer-Rokitansky-Kuster-Hauser (MRKH) syndrome and Morris Syndrome are the most common causes. There is no consensus on the optimal method for the creation of a functional vagina.

Objective: To evaluate and disseminate the experience of neovaginal construction using the modified McIndoeBannister technique.

Method: This is a retrospective descriptive study conducted using medical records of patients submitted to such surgical technique in the period from 2003-2015 at the Assis Chateaubriand University Maternity Hospital of the Federal University of Ceará (MEAC-UFC) in Fortaleza, Ceará, Northeastern Brazil. The technique used consisted of the bilateral removal of skin flaps in leaf format from the inguinal region to avoid exposed scars. Data on age, diagnosis, postsurgical consultations, vaginal examination, complications, and sexual activity.

Results: A total of 23 medical records were analyzed and the patients had a mean age of 21 years old. All patients complained of primary amenorrhea, 18 (78.2\%) had MRKH syndrome and five (21.8\%) presented Morris Syndrome. After surgery, $100 \%$ of patients presented at least a $10 \mathrm{~cm}$ vagina. The most frequent complication in the postoperative period was vaginal vault granulation in seven (30\%) patients. Conclusion: The surgery resulted in a high rate of aesthetic satisfaction and vaginas with width and depth that allowed pleasurable sexual intercourse.
\end{abstract}

Keywords: Vagina; Surgical procedures; sex reassignment surgery.

\section{INTRODUCTION}

The neovagina surgery, that is, the construction of a vagina in patients, can be indicated in cases of absolute or partial absence of the vagina due to congenital abnormalities, trauma, or infection. The surgery is then indicated to enable intercourse [1].

Cases in which the vagina has good elasticity and relative depth should follow expectant treatment, as sexual intercourse and frequent use of molds can lead to a complete enlargement of the vagina. However, in cases of agenesis, surgical treatment is necessary. The most common cause of vaginal agenesis is the congenital absence of the uterus and vagina, known as the Mayer-Rokitansky-KusterHauser Syndrome (MRKH) Syndrome. Another less common cause is the complete androgen insensitivity syndrome (Morris Syndrome). In cases where there is congenital absence of the vagina caused by MRKH Syndrome, the patient may or may not have uterine development, which is generally rudimentary. These malformations occur due agenesis or hypoplasia of the junction of the Müllerian ducts during embryogenesis [2, 3].

In the MRKH Syndrome, the ovaries are morphologically normal, which makes the female pubertal development compatible with karyotype 46,XX. These patients seek medical care complaining of primary amenorrhea between the ages of 15 and 17 years. Vaginal agenesis is the second most 
common cause of primary amenorrhea after gonadal dysgenesis. Its differential diagnosis includes androgen insensitivity, transverse vaginal septum, and imperforate hymen [4].

Another indication for neovagina surgery is the complete androgen insensitivity (Morris Syndrome). It is an X-linked recessive disease in which patients with karyotype 46,XY present female secondary sexual characteristics due to resistance to testosterone resulting from an abnormality in the androgen receptor. These patients typically present female external genitalia and the testes may be palpable (inguinal or pelvic) in the labia majora. These testes end up producing a Müllerian-inhibiting substance, causing regression of the fallopian tubes, uterus and upper third of the vagina. In Morris Syndrome, diagnosis is also given due to primary amenorrhea with normal female pubertal development. It is important to note that the MRKH Syndrome can be differentiated from the Morris Syndrome based on the normal total testosterone level and karyotype (XX in MRKH Syndrome and $\mathrm{XY}$ in Morris Syndrome). Imaging studies (ultrasound and/or MRI) help to clarify the nature of vaginal agenesis and differentiate it from lower transverse septum and imperforate hymen [5].

So far there is no consensus on the optimal method for creating a functional vagina [6]. Treatment involves a multidisciplinary approach with psychosocial support and correction of anatomic abnormality. The psychological aspects involved in the diagnosis of vaginal agenesis, pregnancy failure and, in the case of Morris Syndrome, the presence of XY karyotype may be disappointing for any woman, especially in adolescence [1]. Therefore, surgical treatment should be individualized and result in a vagina that permits intercourse and less scarring as possible.

The first test for neovaginal construction with thin skin graft was performed by Abbé in 1898 and made popular by Mark Indoe. It is still the technique mostly used by gynecologists [7]. This surgery consists in creating a space between the rectum and the bladder, and placing a mold covered with a skin graft taken from the leg or buttock with a dermatome leaving a scar at the site where the graft was removed. With this technique, postoperative dilatation is essential until the woman engages in regular intercourse in order to prevent vaginal stenosis [8].

Within this context, McIndoe-Bannister technique was modified because many women felt uncomfortable with the scars left by the conventional technique. The modified technique consists in the removal of the graft from the inguinal region in order to prevent exposed scars.

Considering the above, the present study aimed to evaluate and disseminate the experience of using the modified McIndoe-Bannister technique in vaginal reconstruction, detailing the procedure performed and the results obtained.

\section{Methodology}

This is a study of retrospective cases carried out using medical records of a census of patients submitted to neovagina surgery using the modified McIndoe-Bannister technique from 2003 to 2015. In all, 37 surgeries were performed during the period analyzed; however, three medical records did not have reports and 11 patients did not return. Therefore, there were only 23 medical records that could be used in the present study. Data such as age, detected pathology, post-surgical return, vaginal examination, early and late complications, and early sexual activity were collected. All the surgeries carried out in the period selected were performed by the same surgeon at the Assis Chateaubriand University Maternity School of the Federal University of Ceará (Maternidade Escola Assis Chateaubriand da Universidade Federal do Ceará - MEAC-UFC) in Fortaleza, Ceará, Northeastern Brazil. The technique used was the same for all patients.

The records are part of demonstrative surgeries for Obstetrics and Gynecology graduate students of the Federal University of Ceará.

Its use for scientific work purposes has been authorized by patients before surgery and was granted by the director of the Assis Chateaubriand University Maternity Hospital of this higher education institution.

The data were recorded in a spreadsheet built on MS Excel for Windows for further analysis.

The technique used is characterized by the following procedures:

1. Removal of a skin flap, in leaf format, bilaterally at the inguinal region. (Image 1)

2. Preparation of the graft - maximum subcutaneous tissue is removed in order to obtain a thin flap. 


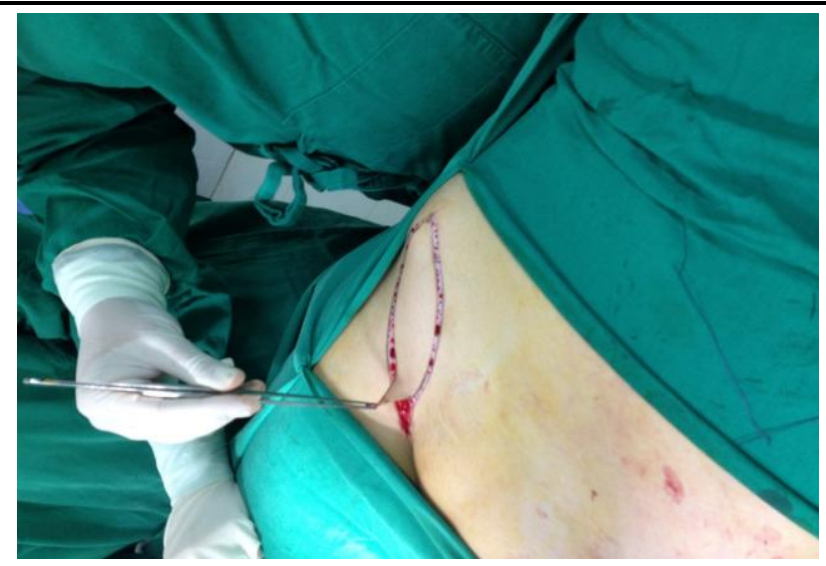

Image1. Removal of a skin flap, in leaf format, bilaterally at the inguinal region

As in the McIndoe-Bannister original technique, the flaps are placed on a rigid acrylic mold and sutured using absorbent material and separate knots. The keratinous side is placed facing the mold to permit that the vascular side attaches and revascularize. Then, a median incision is done in the vaginal introitus creating a tunnel between the bladder and the rectum where the mold is placed.

Postoperatively, the patient remains lying with indwelling urinary catheter and acrylic mold for 8-10 days. After this period, the mold is replaced by another flexible mold made of sponge and condom, which allows the patient to sit and walk.

The complications were analyzed and pain symptoms were classified as: mild, moderate, and severe pain.

At discharge, the patient is instructed to use the flexible mold every day for one month until there is complete healing. After that, the patient must use the mold only at night while sleeping. After two months, the patient is advised to engage in sexual intercourse in order to prevent the narrowing of the vaginal opening.

Outpatient follow-up is carried out at one, three and six months after surgery. After that, the follow-up is carried out annually. Patients receive psychological support in the same institution from diagnosis to postoperative period.

\section{RESUlTS}

We analyzed 23 medical records of patients aged 15-30 years old, with a mean of 21 years $(\mathrm{SD} \pm 4.2)$. All the 23 women who underwent neovagina surgery using the modified McIndoe-Bannister technique had sought the clinic with complaints of primary amenorrhea and were later - after investigation - diagnosed with vaginal agenesis. Eighteen of these women (78.2\%) had the MRKH Syndrome and five (21.8\%) presented Morris Syndrome. All the patients with Morris Syndrome were previously submitted to surgical removal of gonads. Patients' mean number of return consultations for follow-up was 3.2 (SD 1.7), with the first return consultation taking place at 2.2 months (SD 2.4). However, 17 patients (74\%) patients returned for a six-month review.

Regarding satisfaction with sex life, 13 (56.5\%) patients rated it as good, $5(21.7 \%)$ rated it as fair, and $5(21.7 \%)$ records did not contain such information. The most frequent complaint in the immediate postoperative period was moderate pain at the site of the sutures that secure the mold on the vulva. The pain was controlled with nonsteroidal anti-inflammatory drugs and dipyrone.

Some patients have been followed for eleven years and others for only one month. The patients with longer follow-up seek the service when they have any gynecological complaints - most commonly vulvovaginitis - or for a five-year review.

The level of satisfaction of these patients is very good. Dyspareunia during the first intercourse was softened with the use of lubricants and patients did not have more sexual complaints. None complained of vaginal bleeding.

The vaginal examination showed that $100 \%$ of the patients presented good depth and width for penetration considering a midsize vaginal speculum, and the bi digital touch showed that there was at least $10 \mathrm{~cm}$ of vaginal depth. 
The most frequent complication in the late postoperative period was vaginal vault granulation in seven $(30 \%)$ patients, which was treated by performing cauterization with trichloroacetic acid. One patient returned after five years with urethral diverticulum. The patient was referred to the urogynecologist for evaluation and surgery submission.

\section{DisCUSSION}

As the incidence of the MRKH Syndrome (1/4,000-5,000 live births) is greater than Morris Syndrome $(1 / 22,000-99,000)$ [9], a greater number of surgeries for such indication was expected.

The result was satisfactory for the vast majority of patients who returned to the service. They presented pleasurable intercourse and vagina with good depth and width.

Among the advantages of the modified technique, the following should be highlighted:

Unlike the traditional McIndoe-Bannister technique, in which the flap is removed from the buttock or thigh, leaving a scar that causes discontent in patients as it is easily seen, the modified technique uses skin removed from the inguinal area and the scar stays below the underwear or bathing suit, which allows the patient to hide the scar more easily.

It is not necessary to use dermatome for removal of the graft. Therefore, it can be carried out by any gynecologist or surgeon who knows the procedure.

There is no need for abdominal laparotomy or laparoscopy. The is no handling of the colon or rectum, or any other organ that may increase morbidity, as reported by other authors [10,11].

Other techniques such as the laparoscopic Vecchietti procedure [13] and the creation of vaginal pouches from the peritoneum or colon $[9,13]$ are disadvantageous as they are more invasive than the modified McIndoe-Bannister technique. Some studies have reported ulcerative colitis or adenocarcinoma in the segment implanted in the neovagina $[14,15,16]$.

While this procedure quickly achieves the desired result, some techniques take an average of seven years to achieve a good result [17].

There are professionals who use non-surgical treatments such as the Frank's dilators [18]; however, despite being less invasive, it cannot be used in all patients as it requires that the vagina has at least four centimeters. On the other hand, the technique used in the present study can be used in all patients.

\section{CONCLuSion}

Most patients submitted to neovagina surgery using the modified McIndoe-Bannister technique were patients with MRKH Syndrome. They had a mean age of 21 years and complained of primary amenorrhea.

The surgeries resulted in high aesthetic satisfaction and vaginas with good depth and width that allowed pleasurable sexual intercourse.

\section{REFERENCES}

[1] Hecker BR, Mc Guire LS. Psychosocial function in women treated for vaginal agenesis. Am. J. Obstet. Gynecol. 1977; 129(5): 543-7.

[2] ACOG Committee on Adolescent Health Care: ACOG Committee Opinion No. 355: vaginal agenesis: diagnosis, management, and routine care. Obstet. Gynecol. 2006; 108(6): 1605-1609.

[3] Laufer, Mr. Congenital absence of the vagina: in search of the perfect solution. When, and by what technique, should a vagina be created? Current opinion in obstetrics \& gynecology. 2002; 14(5): $441-444$.

[4] Reindollar RH, Byrd JR, McDonough PG. Delayed sexual development: a study of 252 patients. Am J Obstet Gynecol. 1981; 140(4):371.

[5] José E; Landaeta E; Elsa J. Diagnóstico y tratamiento de laamenorrea primaria en adolescentes. a propósito de três casos clínicos. Salus Online. Abril 2011. 15-18.

[6] Guarino N et. al. Vaginoplasty for disorders of sex. Frontiers in endocrinology. março 2013; 4(29): 1-6.

[7] McIndoe AH, Banister JB. An operation for the cure of congenital absence of the vagina. J ObstetGynaecol Br Emp. 1938; 45:490-4. 
[8] Kuohung W, Thompson SR, Laufer MR. Use of acellular human dermal allograft for vaginoplasty in Mayer-Rokitansky-Küster-Hauser syndrome: a case report. J Reprod Med. 2007; 52(9):864.

[9] Khulpateea, BR, David FS. A ten year experience of cecalneovagina procedures for the restoration of sexual function on a gynecology oncology service. Gynecologic oncology. 2014; 134: $150-153$.

[10] Robinson EF, Thomassee MS. Interstitial Ectopic: A Laparoscopic Approach. Journal of Minimally Invasive Gynecology. 2014; 21: S45-S90.

[11] Novier A, Esmat M, Hamza RT. Surgical and Functional Outcomes of Sigmoid Vaginoplasty among Patiens with Variants of Disorders of Sex Development. IntBraz j Urol. 2012; 38: 380-88.

[12] Borruto F, Camoglio FS, Zampieri N, Fedele L. The laparoscopic Vecchietti technique for vaginal agenesis. Int J GynaecolObstet 2007; 98:15.

[13] Davydov, SN. Colpopoiesis from the peritoneum of the uterorectal space. ObstetGynecol 1969; 12:55.

[14] Webster, T et al. Simultaneous development of ulcerative colitis in the colon and sigmoid neovagina. Journal of pediatric surgery; 2013; 48: 669-672.

[15] Alsaleh, A et al. Ulcerative colitis in a neovagina. Case Reports in Internal Medicine; 2014; p89.

[16] Kita, Y et al. Mucinous adenocarcinoma emerging in sigmoid colon neovagina 40 years after its creation: a case report. World journal of surgical oncology; 2015;13: 1.

[17] Morcel K et.al. Sexual and functional results after creation of a neovagina in women with Mayer-Rokitansky-Kuster-Hauser syndrome: a comparison of nonsurgical and surgical procedures. European Journal of Obstetrics \& Gynecology and Reproductive Biology 2013; 169: 317-320.

[18] Frank, RT. The formation of an artificial vagina without operation. Am J Obstet Gynecol 1938; 35:1053. 